\title{
Mismatch-Induced Bit Error Rate in Optical Chaos Communications Using Semiconductor Lasers With Electrooptical Feedback
}

\author{
Y. Chembo Kouomou, Pere Colet, Laurent Larger, and Nicolas Gastaud, Student Member, IEEE
}

\begin{abstract}
We analyze the influence of parameter mismatch between emitter and receiver systems on the bit error rate of an optical chaos communication scheme. Intensity hyperchaos is generated in the system by semiconductor lasers with electrooptical feedback. We find analytical predictions for the dependence of the bit error rate with the amplitude of the various mismatches and the statistical properties of the hyperchaotic carrier. A good concordance is found with numerical and experimental results.
\end{abstract}

Index Terms-Bit error rate (BER), electrooptical feedback, optical chaos communications, semiconductor lasers, synchronization.

\section{INTRODUCTION}

A KEY issue in optical chaos communication schemes [1] is to quantify the detrimental influence of parameter mismatch on the quality of the decrypted message, since the little discrepancies between the emitter and receiver system parameters unavoidably give rise to sustained deviations from the perfect synchronization manifold. In the temporal domain, the related parasite oscillations correspond to the instantaneous synchronization error, and they are sometimes referred to as mismatch noise.

An important characteristic of the mismatch noise is that it not a random noise: it is rather a chaotic noise, i.e., deterministic, even though unpredictable. Generally, this chaotic noise may be divided into three main contributions. The first one is the deviation chaotic noise, which is equal to zero when the emitter and the receiver are identical, and whose amplitude generally grows proportionally to the mismatch [2], [3]. The second contribution is the bursting chaotic noise, whose origin relies on the local unstable invariant sets eventually embedded within the globally stable synchronization manifold [4]. Although this bursting noise vanishes for perfect matching, it increases drastically with the amplitude of the mismatches, and it can be

Manuscript received July 8, 2004; revised September 24, 2004. This work was supported in part by the European Commission through the research project OCCULT under Contract IST-2000-29683, in part by the the MEC (Spain) and FEDER under Projects FIS2004-00953 (CONOCE2), BFM2001-0341-C02-02 (SINFIBIO), and TIC2001-4572-E, and in part by a research grant from the Govern de les Illes Balears.

Y. Chembo Kouomou and P. Colet are with the Instituto Mediterráneo de Estudios Avanzados IMEDEA (CSIC-UIB), Campus Universitat de les Illes Balears, E-07122 Palma de Mallorca, Spain (e-mail: ckyanne@imedea.uib.es; pere@imedea.uib.es).

L. Larger and N. Gastaud are with the GTL-CNRS Telecom, UMR FEMTO-ST 6174, 57070 Metz Cedex, France (e-mail: laurent.larger@univfcomte.fr; ngastaud@georgiatech-metz.fr).

Digital Object Identifier 10.1109/JQE.2004.839686 quite significant even in case of quasi-perfect matching. These two contributions are independent of any message insertion: the first one is associated with system matching accuracy while the second one is associated with synchronization robustness. The third and last contribution, to which little attention has been paid in the literature, is the nonlinear mixing chaotic noise, and it is directly related to the encryption/decryption process. It arises when the chaotic carrier nonlinearly mixes the former two mismatch noise contributions with the message. Consequently, studying the influence of parameter mismatch in a chaos communication scheme requires to take into account five key features: the system itself, the amplitude of the various mismatches, the type of coupling between emitter and receiver, the characteristics of the message, and at last, the encryption/decryption process.

The quality of a digital communication link is almost always evaluated in terms of bit error rate (BER). The issue of BER efficiency in optical chaos communication schemes is quite scarcely addressed in the literature. Some results can be found in [5]-[8]. A more detailed numerical study has been performed by Liu et al. for different laser-chaos communication systems (optical injection, optical feedback, optoelectronic feedback) with various encryption schemes (chaos shift-keying, chaos masking, additive chaos modulation) [9]. In general, the BER depends on several elements such as the random noise in the emitter and receiver systems, dispersion and nonlinear effects in the fiber communication channel, and of course parameter mismatch between the emitter and receiver.

The aim of this work is to study the mismatch-induced BER for an optical communication scheme based on intensity chaos generated by a delayed nonlinear electrooptic oscillator. We first define an appropriate signal-to-noise ratio (SNR) for which we will give an analytical approximation. In a second step we relate this SNR to the BER. We will then compare the theoretical approximations with numerical simulations and experimental results. Finally, this will lead to a discussion on the possible ways to improve the BER of the system.

\section{SYSTEM}

The electrooptical chaos communication scheme under study is represented in Fig. 1. The architecture and principles of the chaos generator are inspired by the pioneering work of Ikeda [10] on nonlinear delay dynamics in Optics, and by the experimental work by Neyer and Voges [11]. The encryption and decryption scheme is detailed in [12], and it corresponds to 


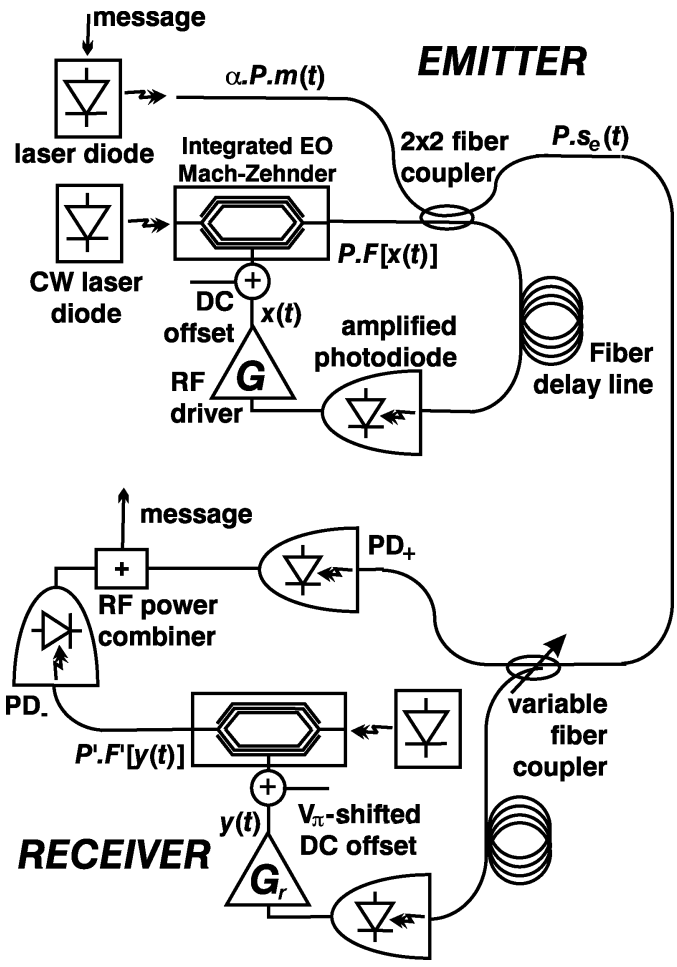

Fig. 1. Experimental setup.

a significant improvement of the setup reported in [13]. The chaotic transmitter consists of a closed electrooptical feedback loop whose main components are the following.

- A $\mathrm{LiNbO}_{3} \quad 12.5-\mathrm{Gb} / \mathrm{s}$ integrated electrooptic Mach-Zehnder (MZ) modulator, for which $V_{\pi}$ is the radio-frequency (RF) half-wave voltage, and $V_{\pi_{\mathrm{dc}}}$ is the bias electrode half-wave voltage. A voltage $V_{B}$ is applied to the bias electrode, and a large amplitude voltage $V(t)$ is applied to the RF electrode to ensure a strong nonlinear dynamical operation of the electrooptic interferometer.

- A continuous-wave (CW) semiconductor laser operating at $1550 \mathrm{~nm}$ and serving as the light source feeding the MZ; its constant optical power is referred to as $P$, and the MZ optical output can thus be written as $P \cos ^{2}\left[\pi V(t) /\left(2 V_{\pi}\right)+\pi V_{B} /\left(2 V_{\pi_{\mathrm{dc}}}\right)\right]$, where $V(t)$ is the electrooptic voltage applied to the MZ.

- A second semiconductor laser with an identical or close wavelength (with respect to the optical spectrum spreading) that is directly modulated by the digital message to be hidden in the chaotic carrier. The 0 bits are assumed to correspond to a null optical power, and the 1 bits have an optical power $\alpha P$. The parameter $\alpha$ can be considered as a measure of the masking efficiency of the message within the chaotic carrier. The binary message light beam can thus be written $\alpha P m(t)$, where $m(t)$ takes the values 0 or 1 .

- A $2 \times 250 / 50$ fiber coupler serving as an all-optical mean for mixing the message within the chaos. The chaos and the message optical beams are assumed to be added in intensity, i.e., without interfering one with each other. This can be ensured, for example, by properly adjusting orthogonal relative polarizations of the two beams in the fiber coupler; note that a fast polarization scrambler should be used before transmission of the chaos + message light beam, to prevent eavesdropping through polarization separation. One output of the fiber coupler is used to transmit the chaos encoded light beam to the receiver, while the other output serves as a feedback signal in the emitter for the generation of the chaotic oscillations.

- A fiber delay-line is used to shift in time the optical signal with a delay $T$.

- An amplified photodiode detects the feedback chaos + message light beam, and converts it into an electrical signal with a sensitivity $A$.

- An RF driver with a gain $G$ is finally used to close the chaotic oscillation loop, applying the previous electrical signal to the RF MZ electrode.

For modeling purposes, the overall normalized electrooptic oscillator gain is expressed as $\beta=\pi A G P /\left(2 V_{\pi}\right)$. It is usually considered as the bifurcation parameter to be tuned in order to observe different dynamical regimes among which the chaotic ones are found. Practical values of the bifurcation parameter $\beta$ vary between 5 and 10 , thus allowing the chaotic regimes required for encryption. These gain values are required for a high amplitude of the electrooptic voltage, thus allowing a highly nonlinear dynamical operation of the MZ interferometer (typically, two to three constructive and destructive interference conditions are scanned dynamically, since $V(t) \gg V_{\pi}$ ). The parameter $\phi=\pi V_{B} /\left(2 V_{\pi_{\mathrm{dc}}}\right)$ corresponds to the operation point of the nonlinear interference transfer function. The normalized dynamical variable proportional to the electrical signal applied to the MZ RF electrode is $x(t)=\pi V(t) /\left(2 V_{\pi}\right)$ (see Fig. 1). The dynamics of the chaotic oscillations is assumed to be ruled by the linear filtering of the electronic feedback, which is modeled by a second-order bandpass filter with a low cutoff characteristic response time $\theta$, and a high cutoff characteristic response time $\tau$. The differential equation ruling the emitter dynamics is thus written as

$$
x+\tau \dot{x}+\frac{1}{\theta} \int_{t_{0}}^{t} x(s) d s=\beta \cdot\{F[x(t-T)]+\alpha m(t-T)\}
$$

where $F[x]=\cos ^{2}(x+\phi)$ is the nonlinear function involved in the dynamical process, and $P \cdot s_{e}(t)$ with $s_{e}(t)=F[x(t)]+$ $\alpha m(t)$ is the transmitted optical signal corresponding to the message superimposed to the chaos. The inloop addition of the message might change significantly the chaotic oscillations with respect to the message-free chaotic oscillations, depending on the actual masking efficiency $\alpha$ used to tune the relative weight of the message compared to the chaos.

The receiver is constructed similarly to the emitter, except it is organized in an open loop architecture. The received optical signal is split first into two branches using a variable fiber coupler. One output of the coupler is directly detected by a fast photodiode, thus giving an electrical signal $K \cdot\left\{\cos ^{2}[x(t)+\right.$ $\phi]+\alpha m(t)\}$, where $K$ is a factor depending on the $\mathrm{CW}$ optical power of the emitter semiconductor laser, on the receiver optical 
splitting ratio, and on the fast photodiode sensitivity. The other coupler output is processed in a similar way than at the emitter after the feedback output of the 50/50 fiber coupler, in order to provide at the receiver a replicated electrical chaos $y(t)$ to be applied to the receiver MZ modulator. This receiver electrical chaos is ruled by

$y+\tau^{\prime} \dot{y}+\frac{1}{\theta^{\prime}} \int_{t_{0}}^{t} y(s) d s=\beta^{\prime} \cdot\left\{F\left[x\left(t-T^{\prime}\right)\right]+\alpha m\left(t-T^{\prime}\right)\right\}$

where $\beta^{\prime}$ depends on the receiver optoelectronic gain between the variable fiber coupler and the MZ modulator electrical input. At the output of the receiver MZ driven by the electrical chaos signal, the photodiode $P D_{-}$generates a chaotic signal corresponding to the locally replicated optical chaos $K^{\prime} \cos ^{2}[y(t)+$ $\left.\phi^{\prime}\right]=K^{\prime} F^{\prime}[y(t)]$, where $K^{\prime}$ depends on the receiver $\mathrm{CW}$ laser power and on the photodiode sensitivity. The latter signal is then added to the direct detection photodiode output $P D_{+}$through a RF power combiner. The power combiner output is thus expressed as

$$
s(t)=K \cdot\{F[x(t)]+\alpha m(t)\}+K^{\prime} F^{\prime}[y(t)] .
$$

The power combiner output achieves chaos cancellation and optimal message retrieving under the following conditions:

- perfect matching $\beta=\beta^{\prime}, T=T^{\prime}, \theta=\theta^{\prime}$, and $\tau=\tau^{\prime}$, leading to the MZ driving signal replication $x(t)=y(t)$;

- adequate biasing of the receiver MZ following $\phi^{\prime}=\phi \pm$ $\pi / 2$, so that $F^{\prime}[y]=1-F[y]$

- proper tuning of the optical power $P^{\prime}$ of the receiver laser, so that $K=K^{\prime}$.

The fulfillment of these three conditions leads to $s(t)=K+$ $K \alpha m(t)$, and the dc value (here equal to $K$ ) is typically canceled by the bandpass filtering of usual high-speed preamplified photodiodes as the ones used in the experiment.

According to this model, we therefore have five control parameters: the time delay $T$, the nonlinear feedback strength $\beta$, the off-set phase $\phi$, the low cutoff response time $\theta$, and the high cutoff response time $\tau$. Note that the primes indicate the receiver parameters, which are in practice always slightly different from the emietter ones.

\section{DETERMINATION OF THE SNR}

The message to be encrypted in this optical chaos communication scheme is a nonreturn-to-zero (NRZ) polar sequence of bits at a fixed bit rate $B$. At the decryption stage in the receiver, the two possible discrete values of this binary signal are 0 for a 0 bit, and $K \alpha$ for a 1 bit. For mathematical commodity, we introduce the signal-to-carrier ratio as $\mu=K \alpha / C$, where $C^{2}=\left\langle\{K F[x(t)]\}^{2}\right\rangle$ is the quadratic average amplitude of the carrier. In fact, $\mu \approx 2 \alpha$ because $\left\langle F^{2}[x(t)]\right\rangle \approx 1 / 2$ in the hyperchaotic regime.

The instantaneous decrypted signal at the power combiner can be decomposed following

$$
\begin{aligned}
s(t) & =K \cdot\left\{\cos ^{2}[x(t)+\phi]+\alpha m(t)\right\}+K^{\prime} \cos ^{2}\left[y(t)+\phi^{\prime}\right] \\
& =s_{0}+\mu C m(t)+n(t)
\end{aligned}
$$

where $s_{0}=\left(K+K^{\prime}\right) / 2$ and

$$
n(t)=\frac{1}{2} K \cos [2 x(t)+2 \phi]+\frac{1}{2} K^{\prime} \cos \left[2 y(t)+2 \phi^{\prime}\right]
$$

is the mismatch noise. It is important to note that in our setup, the mismatch noise $n(t)$ is different from the synchronization error $\epsilon(t)=y(t)-x(t)$, even though $n(t)$ does (like $\epsilon(t)$ ) uniformly vanish to zero in case of perfect matching. For mismatched emitter and receiver, neither the synchronization error nor the mismatch noise are zero.

In the small mismatch approximation, the mismatch noise $n(t)$ can be approximated as

$n(t)=K[\epsilon(t)+\Delta \phi] \sin [2 x(t)+2 \phi]-\frac{1}{2} \Delta K \cos [2 x(t)+2 \phi]$

where $\Delta \phi=\phi^{\prime}-\phi \mp \pi / 2$. From the above equation, the root mean square (rms) amplitude of $n(t)$ can also be approximated as

$$
\left\langle n^{2}\right\rangle \approx \frac{1}{2} K^{2}\left[\left\langle\epsilon^{2}\right\rangle+(\Delta \phi)^{2}+\frac{1}{4}\left(\frac{\Delta K}{K}\right)^{2}\right]=\eta^{2} C^{2}
$$

where $\eta$ is the amplitude of the mismatch noise relatively to the rms amplitude $C$ of the hyperchaotic carrier. We have shown in a former work [14] that for small mismatch $\left\langle\epsilon^{2}\right\rangle=\sigma^{2}\left\langle x^{2}\right\rangle$ with

$$
\begin{aligned}
\sigma^{2}=\frac{1}{3}\left(\frac{\Delta T}{\tau}\right)^{2}+\left(\frac{\Delta \beta}{\beta}\right)^{2}+\left(1-\frac{\pi}{4}\right)\left(\frac{\Delta \tau}{\tau}\right)^{2} \\
-2\left(1-\frac{\pi}{4}\right) \frac{\Delta \beta}{\beta} \frac{\Delta \tau}{\tau}-2\left(1-\frac{\pi}{4}\right) \frac{\Delta T}{\tau} \frac{\Delta \tau}{\tau}
\end{aligned}
$$

where $\Delta p=p^{\prime}-p$ stands for the mismatch related to a given parameter $p$. The rms amplitude $\eta$ of the mismatch noise can, therefore, completely be determined analytically with (7) and (8). It appears that for the physical understanding of the various features of the system, all amplitudes (namely, $\mu, \eta$, and $\sigma$ ) should preferentially be expressed relatively to the rms amplitude $C$ of the hyperchaotic carrier.

From (4), we can consider that the convenient SNR is the peak-to-peak signal-to-rms mismatch noise ratio following

$$
\mathrm{SNR}=\frac{K \alpha}{\sqrt{\left\langle n^{2}\right\rangle}}=\frac{\mu}{\eta} .
$$

Some important remarks can be made at this point. The first one is that there is no bursting mismatch noise contribution in this electrooptical communication scheme. The reason of this absence is that the receiver has a passive (without feedback) nonlinear delayed dynamics, driven by the "chaos + message" optical signal. Therefore, the synchronization manifold is unconditionnaly and uniformly stable. Consequently, neither the signal nor the mismatch noise can destabilize the receiver. The second remark is that the encryption/decryption process we are using does not introduce any nonlinear mixing of the message with the mismatch noise: it only adds them linearly, even though the message is still nonlinearly mixed with the hyperchaotic carrier. This is a key property that allows for an analytical definition of the SNR. Notice that (4) and (5) implicitly assume that the mismatch noise $n(t)$ with or without message remains the same. Equation (9) gives the SNR when no filter is used at the decoding output and, therefore, turns to be independent of the bit rate. 
Therefore, the bit rate of the message could be as large as the bandwidth of the hyperchaotic carrier. If the full chaotic bandwidth is not used, one can practically use a filter at the output, so that the fastest chaotic fluctuations of $n(t)$ could be filtered out without distorting the message, thus improving the SNR. Indeed in that case, (9) provides a lower limit of the SNR. In the general case where a significant amount of mismatch noise is induced by the nonlinear mixing, the SNR cannot be determined as straightforwardly as we did, because of the strong correlation between the noise and the message, and moreover, severe limitation of bit rate is generally observed in these cases.

\section{DETERMINATION OF BER}

We now use the previous results to derive analytically the BER [15]. Generally, each bit is evaluated at a sampling time $t_{s}$ chosen at half of the bit period. Since the binary message is a NRZ polar sequence of bits relatively to its mean value $\mu C / 2$ (which is canceled together with $\left(K+K^{\prime}\right) / 2$ in the process of decryption), the digital decision level system considers that an incoming bit is a 1 if $s\left(t_{s}\right)>0$ and is a 0 otherwise. A 1 bit is mistaken as a 0 when $n\left(t_{s}\right)<-(1 / 2) \mu C$, so that $s\left(t_{s}\right)<0$. Similarly, a 0 bit is mistaken as a 1 when $n\left(t_{s}\right)>(1 / 2) \mu C$. Since a 0 precludes a 1 appearing (and vice-versa), and since both of them have a 1/2 probability of appearance, the BER may be evaluated as the relative sum of the mistaken bits 1 and 0 following

$$
\mathrm{BER}=\frac{1}{2} \operatorname{Prob}\left[n\left(t_{s}\right)<-\frac{\mu C}{2}\right]+\frac{1}{2} \operatorname{Prob}\left[n\left(t_{s}\right)>\frac{\mu C}{2}\right] .
$$

These probabilities can be evaluated from the probability density function (pdf) of the mismatch noise $f_{n}(u)$. It is convenient to scale this pdf with its variance $\eta C$ as

$$
\tilde{f}_{n}(u)=\frac{1}{\eta C} f_{n}\left(\frac{u-\nu}{\eta C}\right)
$$

where $\nu$ is the center value of $f_{n}$. The scaled pdf has exactly the same shape as the original pdf but with zero mean value and variance 1 . Throughout the whole paper, the tilde over a pdf will denote such scaling. From (9) -(11), it can therefore be deduced that

$$
\begin{aligned}
\mathrm{BER} & =\frac{1}{2} \int_{-\infty}^{-(1 / 2)(\mu / \eta)} \tilde{f}_{n}(u) d u+\frac{1}{2} \int_{+(1 / 2)(\mu / \eta)}^{+\infty} \tilde{f}_{n}(u) d u \\
& =\frac{1}{2}\left[1-\int_{-\mathrm{SNR} / 2}^{+\mathrm{SNR} / 2} \tilde{f}_{n}(u) d u\right]
\end{aligned}
$$

The key issue is, therefore, the determination of the pdf $\tilde{f}_{n}$ of the mismatch noise.

It can be seen in Fig. 2(a), the variable $x(t)$ displays a hyperchaotic behavior when the nonlinear feedback gain parameter $\beta$ is high enough, as it is usual for that kind of delayed system [16], [17]. The corresponding pdf is almost Gaussian [Fig. 2(b)], and the main qualitative difference between both is that unlike the Gaussian pdf, the pdf of $x(t)$ does naturally not have infinite
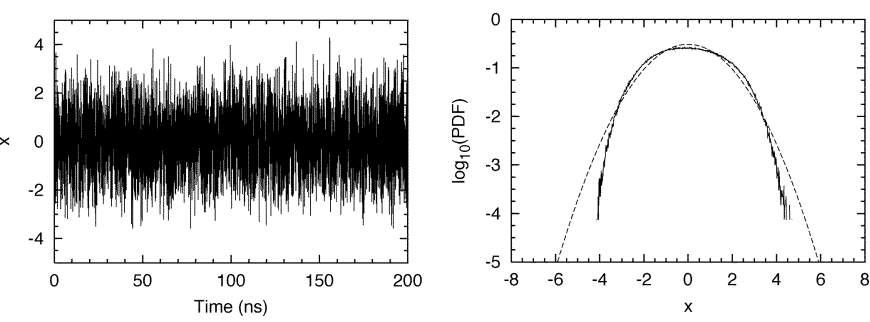

Fig. 2. Numerical simulations of the electrical chaos $x(t)$ and its pdf (solid line). The pdf is resulting from 180000 random samples of the time trace. Its Gaussian counterpart has also represented (dashed line) for comparison. The parameters used for the simulation are $\beta=10, \phi=0.1, T=40 \mathrm{~ns}, \theta=5 \mu \mathrm{s}$, and $\tau=25 \mathrm{ps}$. This set of parameters is compatible with the experimental ones, and it will be used throughout all the paper.
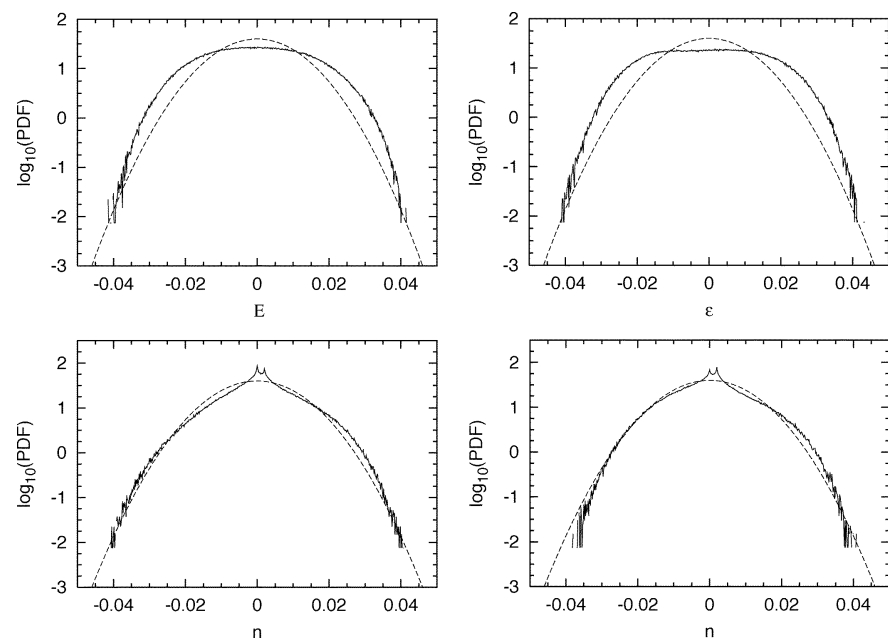

Fig. 3. PDFs for the synchronization error and for the mismatch noise. The upper row corresponds to the pdf of the synchronization error $\epsilon(t)$, while the lower row corresponds to the pdf of the mismatch noise $n(t)$. The left column corresponds to the pdfs for simultaneous $\Delta \beta / \beta=0.005$ and $\Delta \phi=0.004$ mismatches (tuneable mismatches), while the right column corresponds to the pdfs for simultaneous $\Delta \theta / \theta=0.02$ and $\Delta \tau / \tau=0.015$ mismatches (filter mismatches). In all cases, a photodetector mismatch of $\Delta K / K=-0.002$ has been considered (with $K=1.0$ ). The Gaussian counterparts have also be represented in dashed lines. In all cases, the pdfs are the results of 180000 random samples of the related time traces.

tails. Therefore, it can be considered with a good approximation that the scaled pdf of the electrical variable $x(t)$ is very close to its Gaussian counterpart

$$
\tilde{f}_{\text {gau }}(u)=\frac{1}{\sqrt{2 \pi}} e^{-(1 / 2) u^{2}} .
$$

In Fig. 3, the pdfs of the synchronization error $\epsilon(t)$ and of the mismatch noise $n(t)$ have been depicted for various kind of mismatches. We have divided the mismatched parameters into two groups: the so-called "tuneable mismatches" corresponding to $\beta$ and $\phi$ on one hand, and the "filter mismatches" corresponding to the response times $\theta$ and $\tau$ on the other. We have set $\Delta T$ to 0 because the delay-time parameter $T$ can be matched with a relatively high precision at the experimental level. For the synchronization error, it is noticeable that the maxima of the pdfs are significantly lower than the one of their Gaussian counterpart, inducing a noticeable quantitative distortion. On the other hand, for the mismatch noise, one can notice that a better quantitative similitude is observed with the Gaussian, but qualitatively 
important differences can be identified. For example, the pdfs of the mismatch noise are peaky, and the two peaks correspond to the two contributions in (6).

The above analysis indicates that an analytical tool is needed for the comparison of these pdfs relatively to the "reference" Gaussian pdf. The best tool to investigate the effect of the nonGaussianity of a pdf is the Gram-Charlier expansion [18]. Any scaled pdf can be expressed as a function of a Gaussian and its derivatives following the infinite series

$$
\tilde{f}(u)=\tilde{g}(u)+\sum_{k=3}^{+\infty} a_{k} \tilde{g}^{(k)}(u)
$$

with $\tilde{g} \equiv \tilde{f}_{\text {gau }}$. Knowing that

$$
\tilde{g}^{(k)}(u)=(-1)^{k} \operatorname{He}_{k}(u) \tilde{g}(u)
$$

the Gram-Charlier coefficients $a_{k}$ can be recovered through

$$
a_{k}=\frac{(-1)^{k}}{k !} \int_{-\infty}^{+\infty} \operatorname{He}_{k}(u) \tilde{f}(u) d u
$$

where $\mathrm{He}_{k}$ are Hermite polynomials. From a purely mathematical point of view, (16) enables to recover the $a_{k}$ coefficients owing to the orthogonality of Hermite polynomials, as well as Fourier coefficients are recovered owing to the orthogonality of trigonometric functions. In the Gram-Charlier (GC) formalism, any pdf is fully characterized by its $a_{k}$ coefficients, which are uniformly equal to zero in case of a Gaussian pdf. It is interesting to note that for ergodic systems, the GC coefficients are linear combinations of the moments $\int_{-\infty}^{+\infty} u^{k} \tilde{f}(u) d u \equiv\left\langle u^{k}\right\rangle$. Since our hyperchaotic system can be considered as ergodic [14], this property that transforms a complicated spatial integration problem into a simple temporal integration issue will be used later for the GC coefficients computation.

Using both (12) and (14), the BER for a non-Gaussian mismatch noise can, therefore, be evaluated as a function of its GC coefficients following

$$
\mathrm{BER}=\mathrm{BER}_{\mathrm{gau}}(\mathrm{SNR})+\sum_{k=3}^{+\infty} a_{k} \Psi_{k}(\mathrm{SNR})
$$

where

$$
\operatorname{BER}_{\text {gau }}(u)=\frac{1}{2} \operatorname{erfc}\left(\frac{u}{2 \sqrt{2}}\right)
$$

is the BER corresponding to a purely Gaussian noise, and

$$
\Psi_{k}(u)=(-1)^{k+1} 2^{\frac{k-3}{2}} u \frac{\Phi\left[\frac{k+1}{2}, \frac{3}{2} ;-\left(\frac{u}{2 \sqrt{2}}\right)^{2}\right]}{\Gamma\left[\frac{1-k}{2}\right]}
$$

is the $k$ th correction of the Gaussian BER, weighted with the GC coefficient $a_{k}$. It may be interesting to note that the three special functions involved in these equations (the complementary error function erfc, the confluent hypergeometric function $\Phi$, and the

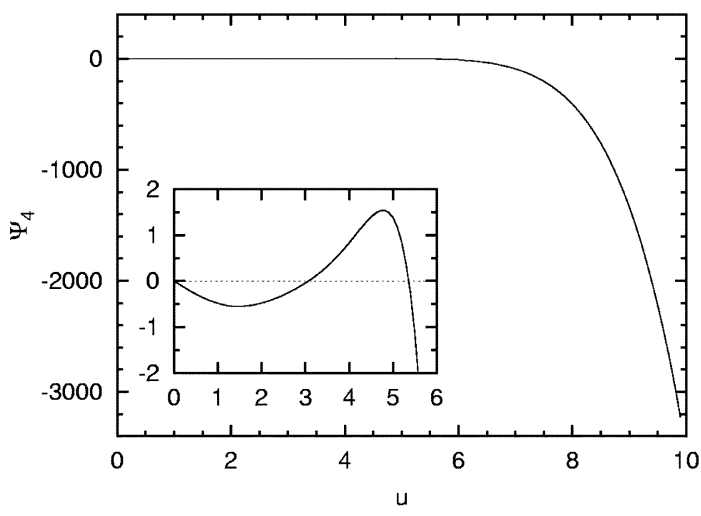

Fig. 4. Representation of the function $\Psi_{4}$. The inset shows a zoom for an argument between 0 and 6 .

complete Gamma function $\Gamma$; see [19]) are usual tools when the BER has to be evaluated analytically in optical and radiofrequency communication systems [20]-[22].

According to (16), the GC coefficients rapidly decrease to zero as $k$ is increased, so that high-order coefficients have less relevance. Hence, only the first coefficients should be considered in a first approximation for the analytical BER evaluation. Let us consider the first odd $\left(a_{3}\right)$ and the first even $\left(a_{4}\right)$ GC coefficients. Typically, $a_{3}$ describes the asymmetry relatively to the central value, and it is considered that noticeable asymmetry is observed when $\left|a_{3}\right|>0.1$, while $\left|a_{3}\right|>0.3$ corresponds to an extreme asymmetry. On the other hand, $a_{4}$ describes the decay to zero of the pdfs tails at infinity, in the sense that $a_{4}<0$ denotes a fast decay to 0 , while $a_{4}>0$ indicates a slow decay to 0 .

The function $\Psi_{k}(u)$ is uniformly equal to zero when $k$ is odd [the denominator in (19) diverges to infinity], so that only the even GC coefficients are involved in the BER evaluation at this precision: in particular, the first corrective term relative to the Gaussian BER-law will be $a_{4} \Psi_{4}$ (SNR). For the mismatch noise $n(t)$, numerical simulations indicate that $a_{4} \sim 10^{-3}>0$, in agreement with Fig. 3(c) and (d) where it can be seen that the pdfs decay slower than the Gaussian to zero. To evaluate the BER correction, we have also computed the function $\Psi_{4}(u)$ in Fig. 4. One can notice that in terms of order of magnitude, no significant deviation relatively to the Gaussian BER law should be expected for SNR $<6$. More precisely, according to the inset of Fig. 4, the BER in the system should be slightly smaller than $\mathrm{BER}_{\text {gau }}$ for $\mathrm{SNR}<3$, and slightly greater that $\mathrm{BER}_{\text {gau }}$ for $3<$ SNR $<5.5$. For SNR $>7$, the corrective term $a_{4} \Psi_{4}$ becomes significant comparatively to $\mathrm{BER}_{\text {gau }}$, so that we should take higher order terms (starting from $a_{6} \Psi_{6}$ ) into account. However, the divergence to infinity of $\Psi_{4}$ indicates that a drastic qualitative change is to be expected for SNR $>7$. This drastic change is in fact a "threshold effect": since the pdf of the noise has finite tails (unlike the Gaussian pdf), there is a limit of the SNR above which the mismatch-induced BER drops to 0 according to (12).

Numerical simulations completely support the above analysis. In Fig. 5, we have numerically evaluated the BER as a function of the SNR for different configurations, varying the bit rate and the origin of the mismatch. The BER is directly measured from the numerical time trace. Two bit rates have been considered, the OC-24 standard bit rate of $1.2 \mathrm{~Gb} / \mathrm{s}$, and the OC-48 standard of $2.5 \mathrm{~Gb} / \mathrm{s}$. As in Fig. 3, we have divided 


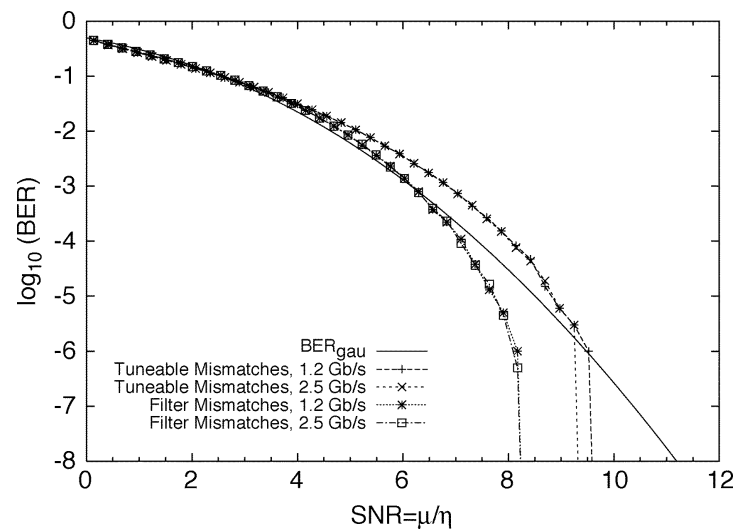

Fig. 5. Numerical simulation for the dependence of the BER with the SNR. Symbols correspond to numerical integration of (1) and (2) with a binary message of $2^{20} \sim 10^{6}$ bits. Tuneable and filter mismatches are considered as in Fig. 3.

the mismatched parameters into two groups, the tuneable mismatches corresponding to $\beta$ and $\phi$ on one hand, and the filter mismatches corresponding to the response times $\theta$ and $\tau$ on the other. For comparison purposes, we have also plotted the theoretical $\mathrm{BER}_{\text {gau }}$ corresponding to a Gaussian pdf.

In agreement with our theoretical analysis, the BER is practically independent of the bit rate, and it does only weakly depend on the origin of the mismatch. As the SNR is increased, the qualitative behavior predicted from the $a_{4} \Psi_{4}(\mathrm{SNR})$ corrective term is effectively observed: from a quantitative point of view, there is no significative difference between the BER computed numerically and the theoretical BER gau. $_{\text {. Qualitatively, the }}$ features predicted analytically are numerically recovered: one can effectively notice that the simulated BER is slightly lower than $\mathrm{BER}_{\text {gau }}$ when $\mathrm{SNR}<3$, and then turns to be slightly higher than $\mathrm{BER}_{\text {gau }}$ until the occurrence of the threshold effect. However, in a realistic situation, even though the mismatch-induced BER may drastically decrease, other sources of BER like random noise or fiber distortions (induced by chromatic dispersion and nonlinearities) may become increasingly important for such high SNR values.

\section{EXPERIMENTAL RESULTS}

In our experimental setup, the electrooptical modulators were commercial $\mathrm{LiNbO}_{3}$ integrated Mach-Zehnder modulators with a dc value of $V_{\pi_{\mathrm{dc}}}=4.0 \mathrm{~V}$, and an RF value (at $1 \mathrm{GHz}$ ) of $V_{\pi}=4.2 \mathrm{~V}$ for $\lambda=1550 \mathrm{~nm}$. One should note that it is the dc value of $V_{\pi}$ that is used to define the off-set phase parameter $\phi$, even though only the RF value is relevant for the hyperchaotic generator. The coherent optical feeders of these modulators were InGaAsP distributed feedback (DFB) semiconductor lasers with a polarization maintaining pigtail, designed for $10-\mathrm{Gb} / \mathrm{s}$ digital system equipments. The delay lines were $8-\mathrm{m}$-long single-mode optical fibers yielding an overall time delay of $40 \mathrm{~ns}$. We also used a matched pair of photodetectors with a gain of $2 \mathrm{~V} / \mathrm{mW}$. The amplification within the nonlinear feedback loops was performed by a pair of RF amplifiers with a gain of $18 \mathrm{~dB}$ and a bandwidth ranging from $30 \mathrm{kHz}$ to $25 \mathrm{GHz}$.

Fig. 6 shows the experimental evolution of the Gram-Charlier coefficients when the power of the emitter feeder laser $P$ is
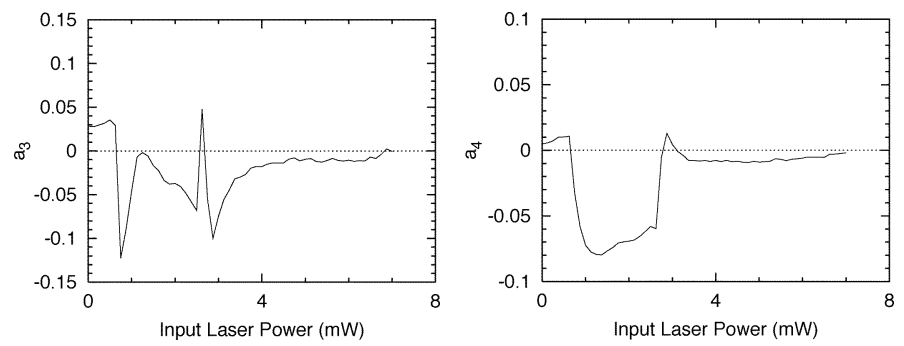

Fig. 6. Experimental evolution of the first odd $\left(a_{3}\right)$ and first even $\left(a_{4}\right)$ Gram-Charlier coefficients corresponding to the electrical variable $x(t)$, when the power $P$ of the emitter feeder semiconductor laser is continuously increased from $0-7 \mathrm{~mW}$ (60000 samples have been used for their computation). Note the convergence to 0 as $P$ is increased.
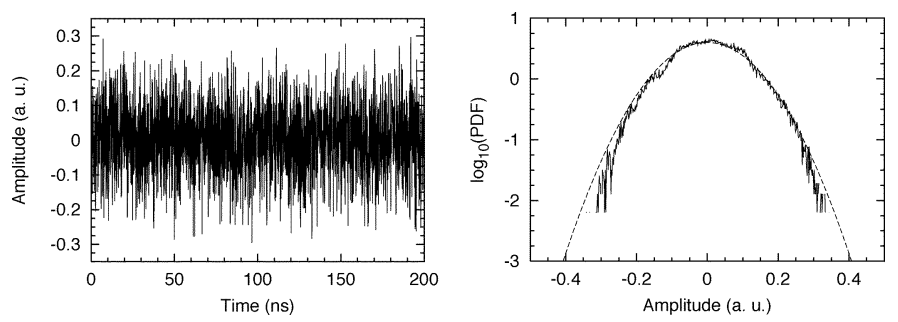

Fig. 7. Experimental time trace of the electrical variable $x(t)$, and its related pdf (without scaling), for a feeder laser power of $P=7 \mathrm{~mW}$. The pdf has been built with 60000 random samples. The Gaussian pdf has also been represented in a dashed line to facilitate the comparison.

continuously increased (note that it corresponds to an increase of $\beta$ ). We have only plotted the first two GC coefficients. It can be observed that for low input power, $\left|a_{3}\right|$ may reach at specific points a noticeable asymmetry, while $a_{4}$ clearly indicates a pdf structure which is quite far from a Gaussian. The system undergoes a sudden change around $P=3 \mathrm{~mW}$ after which the pdf converges to a Gaussian ( $a_{3}$ and $a_{4}$ both converge to zero). The chaotic dynamics is becoming strongly chaotic beyond that point. In an Ikeda system, a similar chaotic behavior has been interpreted as a superposition of quasi-independent processes, leading to a quasi-pdf profile though the central-limit theorem [16].

Fig. 7 displays the electrical hyperchaotic variable used for encryption, as well as its related pdf. It can be noticed in the time trace as well as on the pdf that for high values of the laser power $(7 \mathrm{~mW})$, the hyperchaotic oscillations are practically Gaussian, so that according to the theoretical analysis performed in the previous section, the mismatch noise is also expected to present the same features as in Fig. 3.

Fig. 8 shows the BER experimentally recorded for the 1.2and $2.5-\mathrm{Gb} / \mathrm{s}$ bit rates. The concordance with analytic and numerical results is good, and the analytically predicted qualitative features are recovered. However, some quantitative discrepancies can be noticed above SNR $=7$, probably due to the random noise present in the system.

It should be emphasized that up to SNR values as high as 11, the experimental curves at 1.2 and $2.5 \mathrm{~Gb} / \mathrm{s}$ are quasi-perfectly superimposed, in complete concordance with our theory which states that in first approximation, the bit rate has no influence on the BER variation-law.

Finally, a threshold effect is still experimentally observed: around SNR $=14$, the BER sharply drops to zero, or at least, 


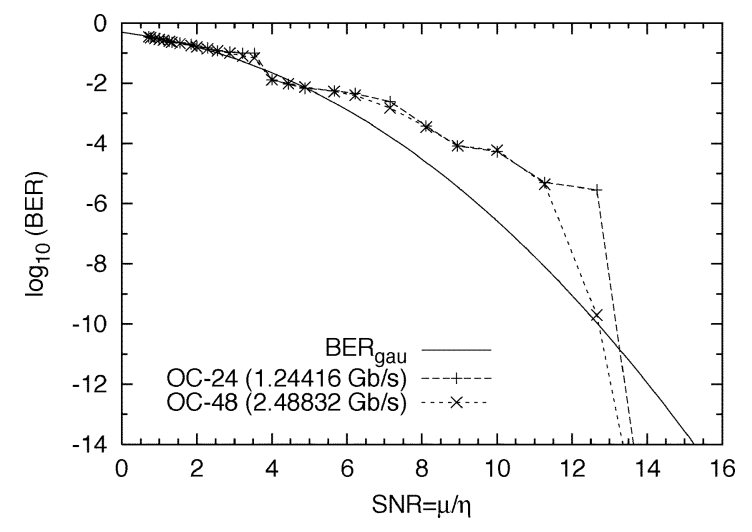

Fig. 8. Experimental variations of the BER at the standard bit rates of OC-24 $(1.24416 \mathrm{~Gb} / \mathrm{s})$ and $\mathrm{OC}-48(2.48832 \mathrm{~Gb} / \mathrm{s})$. The experimental parameters for the OC-24 curve are $P=5 \mathrm{~mW}, V_{B}=0.34 \mathrm{~V}, P^{\prime}=2.88 \mathrm{~mW}$, and $V_{B}^{\prime}=$ $3.42 \mathrm{~V}$. Those of the OC-48 curve are $P=5 \mathrm{~mW}, V_{B}=3.38 \mathrm{~V}, P^{\prime}=$ $2.97 \mathrm{~mW}$, and $V_{B}^{\prime}=7.20 \mathrm{~V}$.

to values that are far below $10^{-15}$. From a more global perspective, one can notice that above SNR $=12$, a small increase of the SNR leads to a very strong improvement of the BER. This limit, therefore, seems to be the target to reach for the fulfillment of the BER requirements in standard optical communication systems. However, for such high SNR values, the message does noticeably deform the pdf of the optical carrier, and thereby induce a faster decay to 0 for the BER.

\section{CONCLUSION}

In this study, we have investigated the influence of the mismatch noise on the BER of an electrooptical chaos communication scheme. We have shown that the pdf of the hyperchaotic carrier and the peak-to-peak-signal-to-rms-noise ratio are the only relevant parameters for the BER evaluation. The non-Gaussianity of the pdf associated to the mismatch noise can be analytically characterized by the Gram-Charlier coefficients, which enable to evaluate the BER of the cryptosystem as a correction of the well-known erfc law corresponding to conventional Gaussian noise.

An interesting issue is to find the way to improve significantly the mismatch-induced BER, or equivalently, the SNR when it is below the threshold value. A first solution would be to decrease as much as possible the value $\eta$ of the rms mismatch noise. This issue has been addressed in a former work [14], and optimal constraints have been derived for the various parameter mismatches in view of the noise level reduction. A second option would be to increase the signal-to-carrier ratio $\mu$, but this solution might threaten the security of the encrypted message, even though along that line, it has yet been shown that an eavesdropper directly tapping the communication channel achieves a BER of only $10^{-2}$ when the authorized chaos receiver achieves a $10^{-9}$ [12]. The two precedent options aim to improve directly the ratio $\mu / \eta$, i.e., the SNR. A third solution, sometimes used in numerical and experimental studies, may be to filter the decrypted signal at the receiver. The design of an optimal filter is not the purpose of this work, even though it seems at first glance that an integrator or an averager synchronized with the clock of the bit rate (to avoid the smoothening of the bit transitions) would be fairly convenient. This work has demonstrated that the pdf of the carrier and the one of the mismatch noise also play a key role in the cryptosystem's performance and that the existence of a threshold value of the SNR above which the BER drops drastically is particularly interesting from a practical standpoint. However, the influence of the various possible pdfs on the security of the communication scheme [23] is an important issue that is still to be investigated.

\section{REFERENCES}

[1] S. Donati and C. R. Mirasso, Eds., "Special issue on optical chaos and applications to cryptography," IEEE J. Quantum Electron., vol. 38, no. 9, Sep. 2002.

[2] R. Brown, N. F. Rulkov, and N. B. Tufillaro, "Synchronization of chaotic systems: the effects of additive noise and drift in the dynamics of the driving," Phys. Rev. E, vol. 50, pp. 4488-4508, 1994.

[3] Y. Chembo Kouomou and P. Woafo, "Stability analysis for the synchronization of semiconductor lasers with ultra-high frequency current modulation," Phys. Lett. A, vol. 308, pp. 381-390, 2003.

[4] J. F. Heagy, T. L. Carroll, and L. M. Pecora, "Desynchronization by periodic orbits," Phys. Rev. E, vol. 52, pp. R1253-R1256, 1995.

[5] H. D. I. Abarbanel, M. B. Kennel, L. Illing, S. Tang, H. F. Chen, and J. M. Liu, "Synchronization and communication using semiconductor lasers with optoelectronic feedback," IEEE J. Quantum Electron., vol. 37, no. 10, pp. 1301-1311, Oct. 2001.

[6] Y. Liu, H. F. Chen, J. M. Liu, P. Davis, and T. Aida, "Communication using synchronization of optical feedback-induced chaos in semiconductor lasers," IEEE Trans. Circuits Syst. I, vol. 48, no. 12, pp. 1460-1484, Dec. 2001.

[7] N. F. Rulkov, M. A. Vorontsov, and L. Illing, "Chaotic free-space laser communication over a turbulent channel," Phys. Rev. Lett., vol. 89, pp. 277905-1-277905-4, 2002.

[8] D. Kanakidis, A. Argyris, and D. Syvridis, "Performance characterization of high-bit-rate optical chaotic communication systems in a back-toback configuration," J. Lightwave Technol., vol. 21, no. 3, pp. 750-758, Mar. 2003.

[9] J. M. Liu, H. F. Chen, and S. Tang, "Synchronized chaotic optical communications at high bit-rates," IEEE J. Quantum Electron., vol. 38, no. 9, pp. 1184-1196, Sep. 2002.

[10] K. Ikeda, "Multiple-valued stationary state and its instability of the transmitted light by a ring cavity system," Opt. Commun., vol. 30, pp. 257-261, 1979.

[11] A. Neyer and E. Voges, "Dynamics of electrooptic bistable devices with delayed feedback," IEEE J. Quantum Electron., vol. 18, no. 11, pp. 2009-2015, Nov. 1982.

[12] N. Gastaud et al., "Electro-optical chaos for multi-10 Gb/s optical transmissions," Electron. Lett., vol. 40, pp. 898-899, 2004.

[13] J.-P. Goedgebuer, P. Levy, L. Larger, C.-C. Chen, and W. T. Rhodes, "Optical communication with synchronized hyperchaos generated electrooptically," IEEE J. Quantum Electron., vol. 38, no. 9, pp. 1178-1183, Sep. 2002.

[14] Y. Chembo Kouomou, P. Colet, N. Gastaud, and L. Larger, "Effect of parameter mismatch on the synchronization of chaotic semiconductor lasers with electrooptical feedback," Phys. Rev. E, vol. 69, pp. 056226-1-056226-15, 2004.

[15] M. Schwartz, Information, Transmission, Modulation and Noise, 4th ed. New York: McGraw-Hill, 1990.

[16] B. Dorizzi, B. Grammaticos, M. Le Berre, Y. Pomeau, E. Ressayre, and A. Tallet, "Statistics and dimension of chaos in differential-delay systems," Phys. Rev. A, vol. 35, pp. 328-339, 1987.

[17] R. Vicente, J. Daudén, P. Colet, and R. Toral, "Analysis and characterization of the hyperchaos generated by a semiconductor laser subjected to a delayed feedback loop," Proc. SPIE, vol. 4986, pp. 452-462, 2003.

[18] R. N. McDonough and A. D. Whalen, Detection of Signals in Noise, 2nd ed. San Diego, CA: Academic, 1995.

[19] I. G. Gradshteyn and I. M. Ryzhik, Table of Integrals, Series and Products, 5th ed. San Diego, CA: Academic, 1994.

[20] Q. T. Zhang, "Probability of error for equal-gain combiners over Rayleigh channels: some closed-form solutions," IEEE Trans. Commun., vol. 45, no. 3, pp. 270-273, Mar. 1997. 
[21] M. K. Simon and M. S. Alouini, "A unified approach to the probability of error for noncoherent and differentially coherent modulations over generalized fading channels," IEEE Trans. Commun., vol. 46, no. 12, pp. 1625-1638, Dec. 1998.

[22] M. A. Najib and V. K. Prabhu, "Analysis of equal-gain diversity with partially coherent fading signals," IEEE Trans. Vehic. Technol., vol. 49, no. 3, pp. 783-791, May 2000.

[23] V. S. Udaltsov, J.-P. Goedgebuer, L. Larger, J.-B. Cuenot, P. Levy, and W. T. Rhodes, "Cracking chaos-based encryption systems ruled by nonlinear time delay differential equations," Phys. Lett. A, vol. 308, pp. $54-60,2003$.

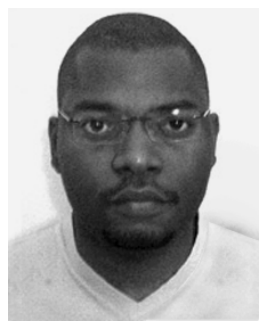

Y. Chembo Kouomou was born in 1976 at Blanc-Mesnil, France. He received the M.Sc. degree in theoretical physics from the University of Yaoundé I, Yaoundé, Cameroon, and the Diploma of Communication Engineering from the National Advanced School of Telecommunications, Yaoundé, both in 2001. He is currently working toward the $\mathrm{Ph} . \mathrm{D}$. degree from the Mediterranean Institute for Advanced Studies, Palma de Mallorca, Spain.

In 2002, he was with the Division of Technical Telecommunication Studies, Minpostel, Cameroon. His research interest involve nonlinear dynamics, optical chaos cryptography, and laser physics.

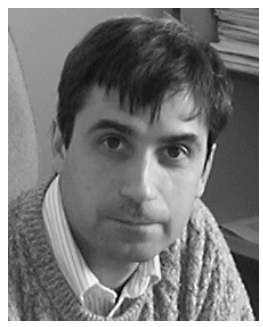

Pere Colet was born in Vilafranca del Penedés, Barcelona, Spain, on April 21, 1964. He received the M.Sc. degree from the Universitat de Barcelona, Barcelona, Spain, and the Ph.D. degree from the Universitat de les Illes Balears, Palma de Mallorca, Spain, in 1987 and 1991, respectively, both in physics.

In 1991, he became a Teaching Assistant with the Departament de Física, Universitat de les Illes Balears. From September 1991 to February 1993 and from April to September 1994, he was a Postdoctoral Fulbright Fellow with the School of Physics, Georgia Institute of Technology, Atlanta. In October 1994, he joined the Departament de Física, Universitat de les Illes Balears. Since May 1995, he has had a permanent research position with the Spanish Consejo Superior de Investigaciones Cientificas. He has coauthored 70 journal papers as well as 20 other scientific publications. His research interests include fluctuations and nonlinear dynamics of semiconductor lasers, synchronization of chaotic lasers and encoded communications, synchronization of coupled nonlinear oscillators, pattern formation and quantum fluctuations in nonlinear optical cavities, and dynamics of localized structures.

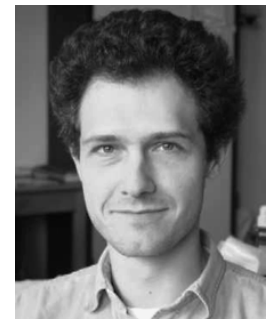

Laurent Larger was born in Colmar, France, in 1968. He received the degree in electronic engineering from the University of Paris XI, Orsay, France, in 1988, and the Agrégation degree in applied physics, the Ph.D. degree in optical engineering, and the Habilitation à Diriger des Recherches from the University of Franche-Comté, Besançon, France, in 1991, 1997, and 2002, respectively.

$\mathrm{He}$ is currently an Associate Professor with the P.M. Duffieux Optics Laboratory, University of Franche-Comté. Since 1998, he has also been involved in the research activities at the GTL-CNRS Telecom Laboratory, the joint research platform between CNRS and the Georgia Institute of Technology, Atlanta. His current research includes the study of chaos in optical and electronic systems for secure communications, delayed nonlinear dynamics, and optical telecommunication systems.

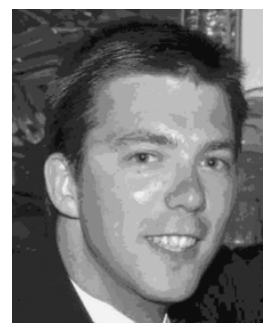

Nicolas Gastaud ( $\mathrm{S}^{\prime} 03$ ) was born in Paris, France, in 1977. He graduated from Saint Jean de Passy, Paris, France, received the B.Sc. degree in engineering (electrical concentration) (with honors) from Trinity College, Hartford, CT, in 2000, the M.Sc. degree in electrical and computer engineering from the Georgia Institute of Technology (Georgia Tech), Atlanta, and the Diplôme de Spécialisation en Traitement et Transmission de l'Information (TTI) from Supélec, Metz, France in 2002. He is currently working toward the Ph.D. degree from the Universite de Franche-Comté, Besançon, France and the Ph.D. degree from Georgia Tech as part of the GTL-CNRS Telecom laboratory in Metz, France.

His research focuses on high-speed chaos encrypted optoelectronic communication systems. 